Original Article

\title{
BINDING STUDIES OF VALGANCICLOVIR TO HUMAN SERUM ALBUMIN BY MULTISPECTROSCOPIC TECHNIQUES
}

\author{
LADE SOMAJI*, RAVI RAPOLU \\ Mewar University, Chittorgarh, Rajasthan, India \\ Email: somunitchem@gmail.com
}

Received: 23 Jun 2018 Revised and Accepted: 03 Sep 2018

\begin{abstract}
Objective: The aim of the present study was to investigate the mode and mechanism of interactions involved towards binding of valganciclovir (VGC) with Human Serum Albumin (HSA) by spectroscopic and molecular modeling studies which can be extrapolated for the ten folds increase of bioavailability over its prodrug galanciclovir.
\end{abstract}

Methods: Herein we employed fluorescence spectroscopy for evaluating the binding constant value, site of interaction and changes in the microenvironment of HSA fluorophores. Circular dichroism (CD) and UV-Visible spectroscopy is used for conformational changes of HSA in the event of binding of valaganciclovir. These experimental studies were further corroborated with molecular modeling studies.

Results: Considerable quenching of fluorescence intensities of fluorophores in the presence of VGC showed that VGC interacts with HSA strongly with a binding constant of $4.11 \times 10^{4} \mathrm{M}^{-1}$ with a free energy change of- $6.26 \mathrm{Kcal} / \mathrm{mol}$. Synchronous fluorescence and CD studies show that the microenvironment and confirmation of HSA are slightly altered in the presence of VGC. Though site marker experiments does not give any clue for identification of site, molecular docking studies showed that VGC binds to site IB of HSA.

Conclusion: The weaker dominant electrostatic interactions with minor contributions of hydrophobic interactions of VGC with HSA at site IB (catalytic domain) might be the probable reason for the relative increase of hydrolysis of VGC to galanciclovir. And moderate binding constant value with HSA implies that HSA can be able to transport VGC under physiological conditions.

Keywords: Valganciclovir, Galancicolvir, Human Serum Albumin, Plasma Proteins, Binding Constants

(C) 2018 The Authors. Published by Innovare Academic Sciences Pvt Ltd. This is an open access article under the CC BY license (http://creativecommons.org/licenses/by/4.0/) DOI: http://dx.doi.org/10.22159/ijpps.2018v10i10.28090

\section{INTRODUCTION}

Diseases that are prevailing and emerging due to infections are rapidly increasing in the present day due to the confluence of socio-economic factors. Among the infections, viral infections form the huge burden to many countries including India. Though few of these viral infections are combated up to some extent, cytomegalovirus (CMV) which belongs to the herpes virus is still at the get-go stage of combat due to lack of complete understanding of the biology of CMV infections. Till date, it is believed that the mode of infection of CMV also follows the modes through which the human immune deficiency virus (HIV) is transmitted. And pity part of the infection is that it causes no symptoms at its initial infection and can only show symptoms during the stage of compromising the immune system (i. e during coinfections). CMV infected patients when co-infected with HIV leads to visual loss. Hence there is an urgent need to develop new antiviral drugs for treating CMV infections. Presently, the medications used for the treatment of CMV is very limited, and among them, galanciclovir $[1,2]$ foscarnet $[2,3]$ or cidofovir $[4,5]$ are routinely used intravenously. Limitations pertaining to the use of these drugs are due to the poor bioavailability and development of resistant by CMV upon prolonged administration of these drugs. In this line, valganciclovir (VGC, fig. 1A.) a prodrug of ganciclovir is developed. VGC is hydrochloride salt, which exists as a mixture of diastereomers, is Lvalyl ester of ganciclovir, which upon oral administration rapidly hydrolysis into active forms of ganciclovir in the presence of hepatic and intestinal esterases. Intracellularly, the active form of galancicovir was further phosphorylated leading to more active form galanciclovir triphosphate, thereby inhibiting the viral DNA replication [6]. The success of VGC over other antiviral drugs in treating CMV is due to its high bioavailability. It was demonstrated that the absolute bioavailability of $\mathrm{CMV}$ is $60 \%$, which are ten folds higher than galanciclovir if administrated orally [7, 8]. This improved efficacy is thought to be due to the presence of valine which mediates the active transportation of VGC to the site of action [9]. This further reiterates that most of the bioavailability of drugs depend on the binding strength towards transporting proteins besides the binding strength towards target sites (proteins/DNA). For many compounds, either administrated orally or intravenously, the first major sight of encountering proteins with the drugs is plasma proteins. Among the plasma proteins, human serum albumin (HSA) provides a terminus in such a way that the drugs will be available beyond their soluble quantities in the plasma.

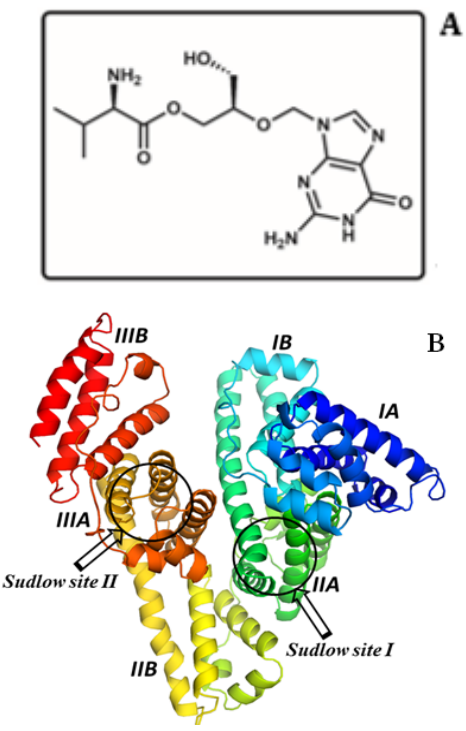

Fig. 1: A) Chemical structure of valganciclovir (VGC) and B) 3D Structure of human serum albumin (HSA) 
HSA a non-glycosylated single polypeptide chain allosteric protein fig. $1 \mathrm{~B}$ consists of 585 amino acids and resembles a heart shape threedimensionally. Structurally, the protein is divided into three domains I (residues 1-195), II (196-383) and III (384-585) with 17 disulfide bridges. HSA is known to bind to a variety of both exogenous and endogenous drugs with high affinities. This high affinity are attributed to its allosteric nature thereby forming an important determinant for evaluating the pharmacokinetic behaviour of many drugs. Since HSA will be synthesized and secreted from liver cells, we presume that the binding nature of VGC towards HSA might alter the hepatic esterase activity of hydrolyzing VGC to its prodrug galancicolvir. To the best of our knowledge, till date, there are no reports wherein the binding properties of VGC towards HSA are studied. Hence in the present study, we aim to study the binding studies of VGC towards HSA by multi spectroscopic and computational studies.

\section{MATERIALS AND METHODS}

\section{Materials}

Valganciclovir (VGC) is procured as a gift sample from Laurel pharma labs, Hyderabad, India. Stock Solution of $1 \mathrm{mmol}$ VGC is prepared in DMSO and further diluted with $100 \mathrm{mmol}$ saline phosphate buffer $(\mathrm{pH}=7.4)$ in order to have a working stock solution of $0.1 \mathrm{mmol}$. Site markers, phenylbutazone (PB) and ibuprofen (IB) are also prepared in a similar manner. HSA (fatty-acid free) is purchased from Sigma Aldrich. The Stock solution of HSA (0.1 mmol) is prepared in saline phosphate buffer $(100 \mathrm{mmol})$ at a $\mathrm{pH}$ of 7.4. All solvents used in the study were of analytical grade.

\section{Methods}

All the working solutions are $1 \mu \mathrm{M}$ of HSA and VGC are titrated from 1 to $10 \mu \mathrm{M}$ for all the experiments performed unless mentioned. The UV-Vis spectra of the VGC-HSA mixtures was monitored over the wavelength range of 220-350 nm using a double beam UV-Vis spectrophotometer (UV-1800Shimadzu ${ }^{\mathrm{TM}}$ ) (Shimadzu Corporation, Tokyo, Japan).

Steady-state fluorescence studies were carried out on Jasco J-815 spectropolarimeter equipped with Peltier thermostat, using a quartz cell of the path length of $1 \mathrm{~cm}$. An Excitation wavelength of $280 \mathrm{~nm}$ is used for exciting both tryptophan and tyrosine, and emission spectra was recorded from 300-500 $\mathrm{nm}$. Excitation and emission bandwidths are set at $5 \mathrm{~nm}$ and $10 \mathrm{~nm}$ respectively. Further, the detector voltage is set at $550 \mathrm{~V}$. All the spectra obtained are the average of three accumulations with a data pitch of $1 \mathrm{~nm}$ and a scan speed of $100 \mathrm{~nm} / \mathrm{min}$. In order to reduce the probable absorption of emitted light by VGC, which affects the emitted light (inner filter effect), the obtained fluorescence spectra were corrected for the inner filter effect by the following equation.

$$
\mathrm{F}_{\text {Cor }}=\mathrm{F}_{\text {obs }} \times \mathrm{e}^{(\text {Aexi+Aemi }) / 2}
$$

Wherein $\mathrm{F}_{\text {cor }}$ and $\mathrm{F}_{\text {obs }}$ are the corrected and observed fluorescence intensities respectively, and $A_{\text {exi }}$ and $A_{\text {emi }}$ are the respective absorbance intensities of excitation and emission wavelengths of HSA protein. Site identification studies were also performed under similar conditions. For temperature studies, the cell temperature of 273, 293 and $303{ }^{\circ} \mathrm{K}$ is maintained using nitrogen gas under abovementioned conditions. Synchronous experiments $(\Delta \lambda=15$ and $\Delta \lambda=60$ ) were carried on LS55 spectrofluorometer, PerkinElmer corporate, USA with an excitation and emission slit widths of $8 \mathrm{~nm}$ and $10 \mathrm{~nm}$ respectively with a scan speed of $100 \mathrm{~nm} / \mathrm{min}$ over the wavelength range of 200-350 $\mathrm{nm}$. The spectra obtained are the average of three scans.

CD measurements were also carried on the above instrument (Jasco J-815 spectropolarimeter) using a quartz cell length of $0.2 \mathrm{~cm}$ in the far UV range (190-300 $\mathrm{nm}$ ) at room temperature. CDNN 2.1 software is used for evaluation of structural changes.

Molecular modelling studies were performed using autodock 4.2 program using a lamarckian genetic algorithm. HSA X-ray crystal structure is taken from brookhaven protein data bank (PDB ID: 1A06) and the protein topology was prepared using autodock 4.2 . PBD structure of valganciclovir is obtained from https://www.
drugbank.ca/drugs/DB01610 and is used without further modifications. Grid box of dimensions $126 \times 126 \times 126$ was set with a grid spacing of $0.703 \mathrm{~A}^{\circ}$. And the center of the grid was set to 2.95, 3.18 and $2.35 \mathrm{~nm}$. Docking parameters were set at default settings, and 25 conformations were run using a genetic algorithm. The conformer with the lowest binding energy that corresponds to the experimental value is visualized and analyzed using PyMol software.

\section{RESULTS AND DISCUSSION}

\section{UV-visible analysis}

In an initial attempt, we employed UV-Visible spectroscopy towards understanding the binding interactions of HSA with VGC. From fig. 2 it is evident that HSA showed two significant absorption peaks at 200-210 $\mathrm{nm}$ and 260-300 $\mathrm{nm}$ which are fingerprint regions for the predicting secondary structural changes and the microenvironment of intrinsic chromophores respectively. Sequential addition of $1 \mu \mathrm{Mof}$ VGC to the final concentration of $10 \mu \mathrm{M}$ of VGC leads to a gradual increase in the intensity of the absorption peak at $280 \mathrm{~nm}$. Though the increase is very marginal these are significant for understanding the changes in the microenvironment of the aromatic residues. Secondly, there is a marginal red shift in $200-210 \mathrm{~nm}$ along with the increase in the intensity of the absorption. This result suggests that the polarity of aromatic amino acid residues, particularly trp-214, protein backbone conformation and $\alpha$-helix content of HSA might be slightly altered with the addition of VGC. To further elucidate and to affirm structural changes of HSA during interaction with VGC, Circular dichroism (CD) studies were employed.

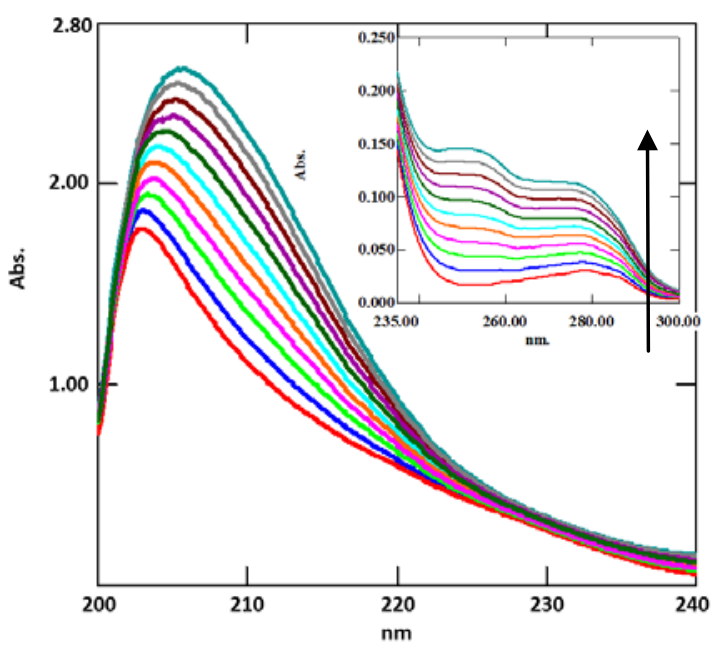

Fig. 2: UV-visible spectra of HSA $(1 \mu \mathrm{M})$ with varying concentrations of VGC $(1-10 \mu \mathrm{M})$

\section{Circular dichroism analysis}

CD spectral analysis of HSA showed two characteristic negative peaks at 208 and $222 \mathrm{~nm}$ that corresponds to $\alpha$-helix content of the native protein. Sequential addition of $1 \mu \mathrm{M}$ of VGC to HSA resulted in the increase of the intensity of these two peaks fig. 3A. However, such an increase in intensity is very marginal indicating that the secondary structural elements of HSA are well preserved during the interaction with VGC thereby not affecting the transporting capability of HSA. Similar observations were made in the recent studies, wherein the $\alpha$-helical content of HSA is reduced to a lesser extent during interaction with drugs $[10,11]$. Further, the extent of decrease in the $\alpha$-helical content is quantified. The percentage of $\alpha$ helix in the native state is found to be $\sim 67.9 \%$ and gradually decreased to $\sim 63 \%$ while $\beta$-sheets and random coils are increased from 18.1 to $20 \%, 14.8$ to $18.3 \%$ respectively during the course of VGC titrations fig. 3B. This result indicates that the microenvironment of the protein is changed and the compactness of the protein is reduced during the interaction with VGC. These observations further corroborate with that of UV spectral analysis. 


\section{$\%$ of $\alpha$-Helix Content}
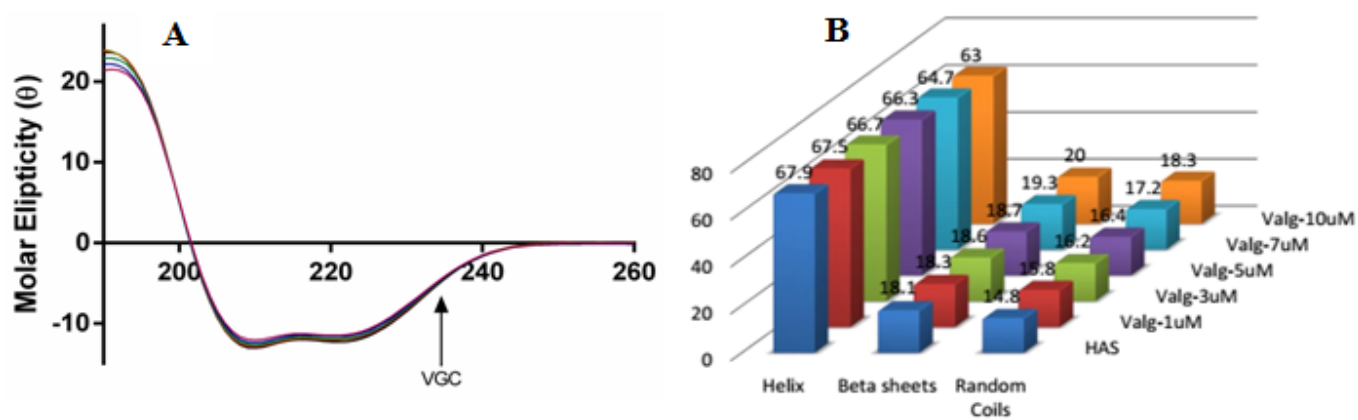

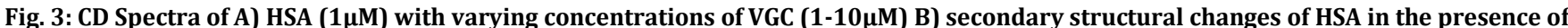
$\operatorname{VGC}(1-10 \mu M)$

\section{Fluorescence spectroscopic analysis}

\section{Synchronous fluorescence}

In addition to UV-Visible spectroscopy and CD analysis, an alternative approach to ascertain the changes in the microenvironment of fluorophore residues of proteins during binding with the drugs is to study synchronous fluorescence behavior of fluorophores. Synchronous fluorescence in its simplest form is a way of scanning emission and excitation monochromators simultaneously with the fixed interval of wavelengths $(\Delta \lambda=15 \mathrm{~nm}$ for tyrosine and $\Delta \lambda=60 \mathrm{~nm}$ for tryptophan). Possible shifts in the emission maxima reveal the hydrophobicity of fluorophores environment [12-14]. Accordingly, in the present study synchronous fluorescence spectra at a fixed wavelength intervals of $15 \mathrm{~nm}$ and $60 \mathrm{~nm}$ were studied. Fig. 4A shows that there are no considerable shifts in emission maxima at $\sim 285 \mathrm{~nm}$ which imply that the microenvironment of tyrosine residues are not altered much due to binding of VGC with HSA. However, synchronous fluorescence spectra at a fixed wavelength interval of $60 \mathrm{~nm}$ fig. $4 \mathrm{~B}$ showed a marginal blue shift of $\sim 3 \mathrm{~nm}$ indicating that the microenvironment of tryptophan residue is slightly altered due to the presence of VGC in the close proximity. Hence these results are consistent with those of UV and CD spectral studies.
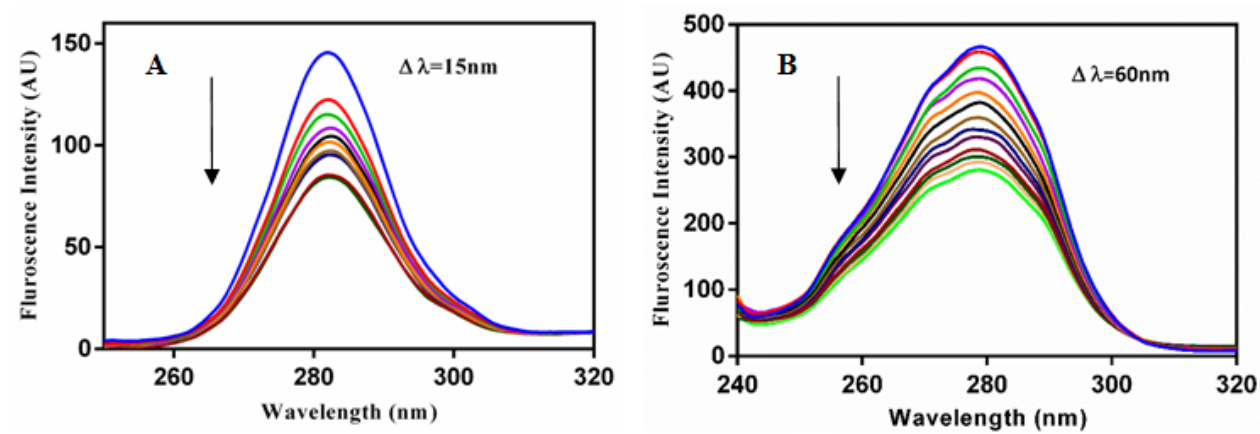

Fig. 4: Synchronous spectra of VGC with HSA A) $\Delta \lambda=15 \mathrm{~nm}$ and B) $\Delta \lambda=60 \mathrm{~nm}$ with increasing concentrations of VGC (1-10 $\mu \mathrm{M})$

\section{Quenching mechanism and binding constant evaluation}

Besides the above techniques that were mentioned and discussed, fluorescence spectroscopy provides an excellent platform for screening and understanding the nature of binding interactions between protein and small molecules by taking advantage of intrinsic fluorophores such as tryptophan, tyrosine, and phenylalanine. Presence of single tryptophan residue in the given protein is fairly enough for performing fluorescence since it is having high quantum yield when compared to other amino acids. In addition to understanding the binding interactions, binding constants, binding sites, and the effect of temperature on the binding mode can also be evaluated with the aid of this technique. Hence in the present study, we adopted and employed this technique for further screening of binding interactions of HSA with VGC.

Titration of VGC with HSA in increments of $1 \mu \mathrm{M}$ resulted in a sequential decrease of fluorescence intensity at $340 \mathrm{~nm}$ fig. 5 . Without considerable shifts in emission maximum and/or emission peak shapes. Such observations indicate that interactions exist between VGC and HSA and compliments with those of the results that are obtained from UV-Visible studies and conformational analysis. Further, this gradual decrease of fluorescence intensity or quenching suggests that VGC is interacting with HSA at the site, which is at the vicinity of trp-214.

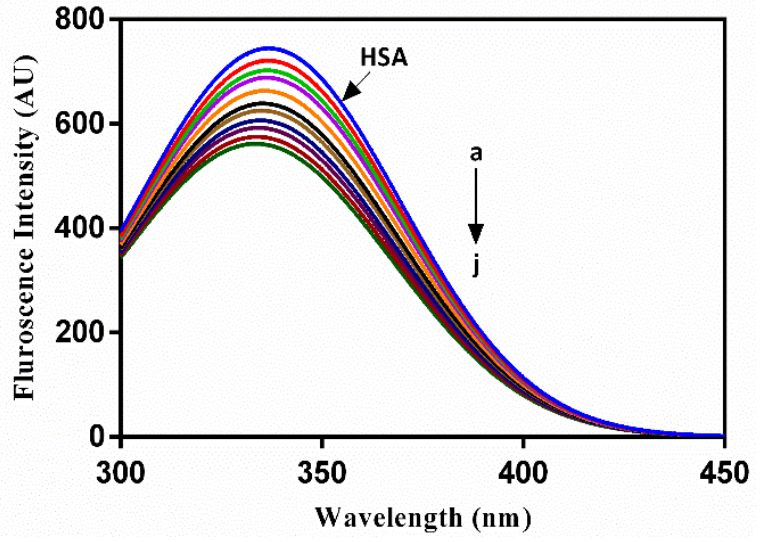

Fig. 5: Fluorescence spectra of HSA in the presence of increasing concentrations of VGC $(1-10 \mu \mathrm{M})$

It is evident from the literature [15-17] that the process of quenching takes place via either through statically or dynamically during protein-drug interactions. Static quenching process can be 
differentiated from dynamic quenching process by examining the quenching rate constants at different temperatures [18] or by calculating the lifetime of the fluorophore in the excited states. Towards understanding the quenching mechanism involved during the interaction of VGC with HSA we applied the well-known Sternvolmer equation as below.

$$
\mathrm{F}_{\mathrm{o}} / \mathrm{F}=1+\mathrm{K}_{\mathrm{q}} \tau_{0}[\mathrm{Q}]=1+\mathrm{K}_{\mathrm{sv}}[\mathrm{Q}]
$$

Where, $\mathrm{F}_{\mathrm{o}}$ and $\mathrm{F}$ are fluorescence intensities of fluorophore in the absence and presence of $\mathrm{VGC},[\mathrm{Q}]$ is concentration of quencher in this case it is $[\mathrm{VGC}], \mathrm{k}_{\mathrm{q}}$ is bimolecular quenching rate constant, $\tau_{0}$ lifetime of the fluorophore $\left(\sim 10^{-8} \mathrm{~s}\right)$ in the absence of VGC and $\mathrm{K}_{\mathrm{sv}}$ is Stern-volmer quenching constant and can be obtained as given below

$\mathrm{K}_{\mathrm{q}}=\mathrm{K}_{\mathrm{sv}} / \tau_{0}$.

From the plot of $\mathrm{F}_{\mathrm{o}} / \mathrm{F}$ vs [VGC] the stern-volmer quenching constant was found to be $3.4 \times 10^{12} \mathrm{M}^{-1} \mathrm{~s}^{-1}$ and is far larger than maximum diffusion collisional quenching rate constant for various quenchers in the presence of biopolymers $2.0 \times 10^{10} \mathrm{M}^{-1} \mathrm{~S}^{-1}$.

Table 1: Stern-volmer (Ksv), quenching constant $(\mathrm{Kq})$, binding constant $\left(\mathrm{K}_{\mathrm{b}}\right)$ and free energy $\left(\Delta \mathrm{G}^{\circ}\right)$ of $\mathrm{VGC} \mathrm{HAS}$ complex at 293 and $303{ }^{\circ} \mathrm{K}$

\begin{tabular}{lll}
\hline $\mathbf{T}\left({ }^{\circ} \mathbf{K}\right)$ & $\mathbf{2 9 3}^{\circ} \mathbf{K}$ & $303^{\circ} \mathbf{K}$ \\
\hline Ksv x $10^{4}\left(\mathrm{M}^{-1}\right)$ & 3.51 & 6.38 \\
$\mathrm{Kq} 10^{12}\left(\mathrm{M}^{-1} \cdot \mathrm{s}^{-1}\right)$ & 3.51 & 6.38 \\
$\mathrm{R}^{2}$ & 0.99 & 0.98 \\
$\mathrm{~K}_{\mathrm{b}} 10^{4}\left(\mathrm{M}^{-1}\right)$ & 3.95 & 3.32 \\
$\operatorname{Binding~sites~(n)~}$ & 1.02 & 0.87 \\
$\mathrm{R}^{2}$ & 0.99 & 0.96 \\
$\Delta \mathrm{G}^{\circ}(\mathrm{Kcal} / \mathrm{mol})$ & -6.24 & -6.14 \\
\hline
\end{tabular}

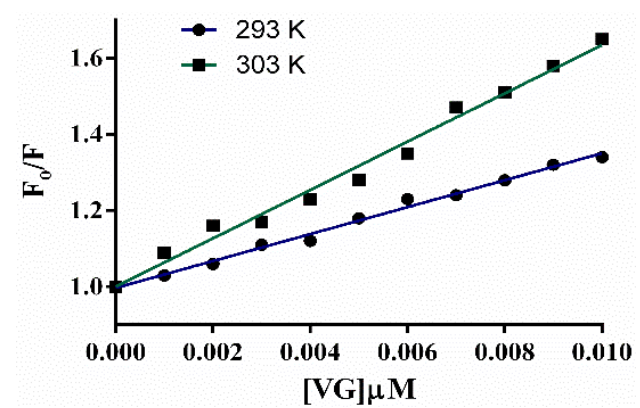

Fig. 6: Stern-volmer plots for VGC-HSA complex at 293 and $303^{\circ} \mathrm{K}$

Therefore the interaction of VGC with HSA follows a static quenching mechanism [19]. In order to further ascertain this quantitatively, stern-volmer constants were evaluated at two different temperatures $\left(293{ }^{\circ} \mathrm{K}\right.$ and $\left.303{ }^{\circ} \mathrm{K}\right)$. From table 1 and fig. 6 it is evident that there is an increase in the stern-volmer constants with the increase in temperature, which demonstrates that the mechanism involved in the formation of HSA-VGC complex is due to dynamic collision rather than complex formation. This is in contrary to that of lifetime measurements of fluorophores. The mechanism of static and/or dynamic complex formation can be further confirmed by taking the difference in the absorption spectra of HSA-VGC complex and the VGC absorption [20]. Since static quenching is the result of the complex formation of the HSA-VGC in the ground state, the difference in the absorption spectra near ultraviolet region (250$400 \mathrm{~nm}$ ) of HSA-VGC complex and VGC is significant. This result further illustrates that the quenching mechanism involved in the HSA-VGC complex is by static quenching (data not shown).

For evaluating the binding strength and number of binding sites, the modified stern-volmer equation is used.

$\log \left(\mathrm{F}_{0}-\mathrm{F}\right) / \mathrm{F}=\log \mathrm{K}+\mathrm{n} \log [\mathrm{Q}]$

Where $\mathrm{K}, \mathrm{n}$ and $[\mathrm{Q}]$ represents binding constant, number of binding sites and concentration of VGC respectively. From double reciprocal log plots, the binding constant is found to be $4.11 \times 10^{4} \mathrm{M}^{-1}$ and the number of binding sites is found to be 1.0. This data indicates that VGC and HSA form a moderately strong complex with one binding site. Since the complex formation is driven by intermolecular forces such as van der Waals, hydrogen bonds, hydrophobic forces and electrostatic forces which contribute to the free energy change for the complex formation hence standard free energy for the complex formation is calculated by the following equation.

$\Delta \mathrm{G}^{\circ}=-\mathrm{RT} \ln \mathrm{K}$
Where $\Delta \mathrm{G}^{\circ}$ is free energy change, $\mathrm{R}$ is the gas constant and $\mathrm{K}$ is the binding constant of VGC at temperature $\mathrm{T}{ }^{\circ} \mathrm{K}$. The calculated free energy change is found to be $-6.27 \mathrm{Kcal} / \mathrm{mol}$ during the interaction of VGC with HSA and the negative sign implies that the complex formation is spontaneous wherein hydrogen bonding and hydrophobic interactions play a major role during complex formation.

\section{Nature of binding forces involved in binding mode}

Non-covalent interactions, namely Van derWaals forces, electrostatic forces, hydrophobic interactions and hydrogen bonds are the principal driving forces that are involved during complex formation between protein and drug. Depending on the nature of functional groups present in the drug, aforementioned forces dominate over other and contribute to thermodynamic parameters such as enthalpy $(\Delta \mathrm{H})$ and entropy $(\Delta \mathrm{S})$ changes during complex formation [20]. Hence for further elucidating these driving forces that are involved in complex formation between VGC and HSA thermodynamic parameters were calculated using Van't Hoff equation which is given as below.

InK $=-\Delta H^{\circ} / \mathrm{RT}+\Delta \mathrm{S}^{\circ} / \mathrm{R}$

$\Delta \mathrm{G}^{\circ}=\Delta \mathrm{H}^{\circ}-\mathrm{T} \Delta \mathrm{S}^{\circ}$

Where $\mathrm{K}$ is the binding constant at the temperature $\mathrm{T}$ and $\mathrm{R}$ is the gas constant and the values of $\Delta \mathrm{H}^{\circ}$ and $\Delta \mathrm{S}^{\circ}$ were evaluated from the slope and intercept of the plots of $\ln \mathrm{K}$ against $1 / \mathrm{T}$. Thermodynamic parameters at temperatures 293 and $303{ }^{\circ} \mathrm{K}$ were evaluated and found that a small negative value $(-12.82 \mathrm{~kJ} / \mathrm{mol})$ of enthalpy and positive value $(44.22 \mathrm{~J} / \mathrm{mol} \mathrm{K})$ of entropy were associated with the complex formation between VGC and HSA, which implies that complex formation between HSA and VGCis driven by electrostatic interactions. However, the contributions of hydrophobic interactions for positive entropy cannot be ignored [24]. The negative values of $\Delta \mathrm{G}^{\circ}$ at the studied temperatures (-6.24 and-6.14 $\mathrm{Kcal} /$ mole, respectively, table 1 ) also indicates the complex formation is spontaneous with the large contribution of entropy and with small contribution from enthalpy.

\section{Binding site identification}

Being allosteric in nature, HSA possesses more than one binding site and therefore forms a major depot for most of the exogenous and endogenous molecules. However, there are two primary drug binding sites namely site I (Sudlow site I) and site II (Sudlow site II) located in subdomains IIA and IIIA respectively. Besides these primary binding sites recently it was demonstrated that subdomain IB functions as the secondary binding site[22]. In order to identify the probable binding site of VGC with HSA, site displacement method was adopted [23]. Among the known site markers of HSA, 
phenylbutazone $(\mathrm{Pbz})$ and ibuprofen $(\mathrm{Ibu})$ were used in the present study. Complexes of HSA-Pbz and HSA-Ibu are prepared by mixing $1 \mu \mathrm{M}$ each and incubated for $5 \mathrm{~min}$ before titrating with VGC in increments of $1 \mu \mathrm{M}$. From fig. 7A it is evident that the fluorescence intensity of HSA-Pbz complex gradually decreased by the addition of VGC without considerable blue/red shifts and the binding constant for VGC in the presence of $\mathrm{Pbz}$ is found to be $1.20 \times 10^{4} \mathrm{M}^{-1}$. Such a decrease in the binding constant value implies that the VGC might be approaching near to the Pbz binding site i. e Sudlow site I. On the other hand, the addition of VGC to the complex of HSA-Ibu complex fig. 7B, there is also a gradual decrease in the fluorescence intensity, but the amount of quenching is relatively less in comparison to that of HSA-Pbz complex. And the binding constant value VGC is found to be $8.14 \times 10^{4} \mathrm{M}^{-1}$ in the presence of Ibu site marker. This implies that VGC might not be approaching the Ibu binding site i. e Sudlow site II [24]. Therefore, in order to further elucidate and confirm the binding site the complementary approach of molecular modeling is applied [25].
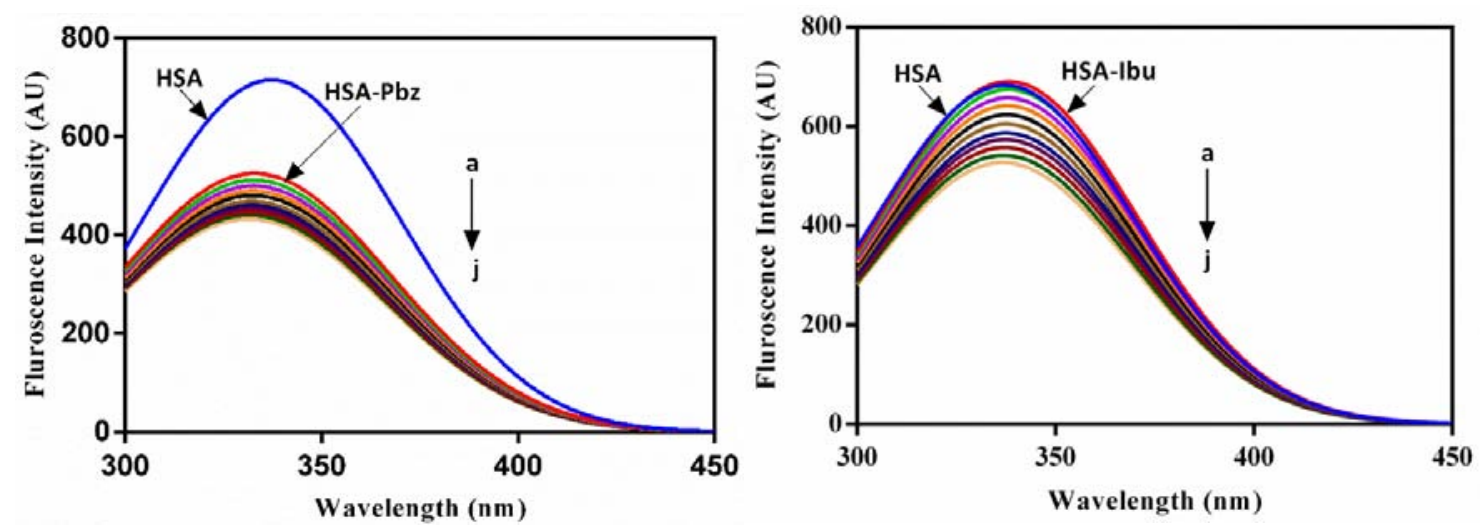

Fig. 7: Fluorescence spectra of VGC-HSA in the presence of site markers A) Phenylbutazone (Pbz) and B) Ibuprofen (Ibu)

\section{Molecular docking analysis}

Molecular docking approach provides complementary results that ameliorate the understanding of molecular interactions involved in the complex formation of HSA with VGC. Making use of this approach, twenty-five conformations of HSA-VGC complexes were generated in insilico conditions. Among the generated conformations, seven conformers are found to interact with site IB and are mostly buried in the groove while six conformers are found to interact with site IIIB, however, having high free energy when compared to the conformers that are bound to site IB. Further, only one conformer is found to bind to site IIA of HSA this reveals that VGC is not exactly competing for the site I but approaching to the site near to site I which is also in accordance with the decrease in the binding constant values. Furthermore, the free energy change $(-5.32$
$\mathrm{Kcal} / \mathrm{mole})$ is close to the experimental values $(-6.27 \mathrm{Kcal} / \mathrm{mol})$ that are obtained from fluorescence. Therefore, among the seven conformers that are found to interact with the site IB the best docked stable conformer is subjected for further analysis. From fig. 8 it is evident that VGC forms four hydrogen bonds with Try-161, Phe134, His-146 and Arg-186 with the bond distances of 2.9, 3.3, 2.2 and $3.3 \mathrm{~A}^{\circ}$ respectively which are at a favourable distance for formation strong hydrogen bonding. Further, the guanosine group of VGC is further stabilized by hydrophobic interactions with Lys-190, Ile-142, Leu-115, and Arg-186 whereas the ester functionality is interacting with Pro-118, Phe-134 and Met-123 hydrophobically within the distance of $4.2-5.5 \mathrm{~A}{ }^{\circ}$. These results indicate that VGC is stabilized by dominant electrostatic interactions with little contributions of both hydrophobic and hydrophilic interactions which complement the results that are obtained from thermodynamic studies.

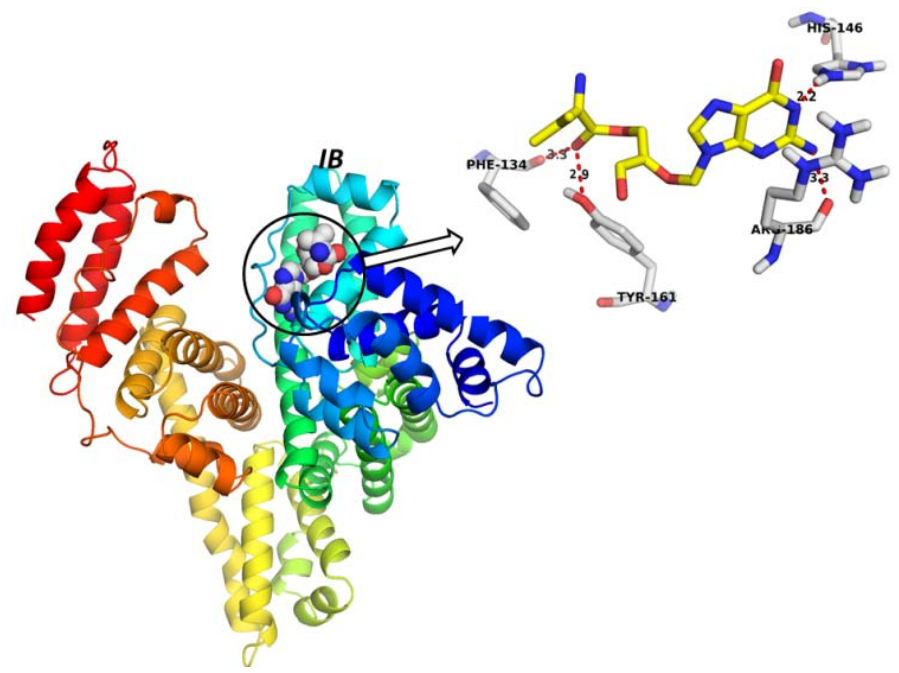

Fig. 8: Schematic view of lowest binding energy docked conformer of VGC with HSA. Zoomed view showing hydrogen bonding distances along with interacting amino acids 


\section{CONCLUSION}

In the present study, we explored the binding interactions of VGC with HSA by spectroscopic and molecular modelling studies. A Slight red shift in UV-Visible analysis and minor/no changes in the synchronous fluorescence demonstrated that the microenvironment of HSA was changed marginally during interaction with HSA. Small changes in alpha-helical content by $\mathrm{CD}$ analysis further confirm this. Moreover, fluorescence quenching studies illustrate that complex formation takes place through a static quenching mechanism with a binding constant of $4.11 \times 10^{4} \mathrm{M}^{-1}$. Temperature studies indicate that electrostatic interactions and hydrogen bonding are the primary forces responsible for complex formation with minor contributions of hydrophobic interactions. Binding site identification by fluorescence indicate that the VGC is binding near to the site IIA (Sudlow site I), however, insilico studies reveals that the binding site for VGC is site IB since the conformers that bind to site IB is having more free energy change when compared to the conformers that bind to site IIIB. And the resulted conformers are stabilized by four hydrogen bonds with hydrophobic contributions. This preferential binding of VGC at Site IB, a catalytic subdomain of HSA might increase the rate of hydrolysis of VGC to its prodrug galanciclovir thereby increasing the bioavailability of VGC.

\section{ACKNOWLEDGMENT}

None

\section{SUPPLEMENTARY MATERIAL}

None

\section{AUTHORS CONTRIBUTIONS}

All the work is done by Somaji Lade and Ravi Rapolu has read and corrected the manuscript.

\section{CONFLICTS OF INTERESTS}

\section{Declared none}

\section{REFERENCES}

1. Spector SA, Weingeist T, Pollard RB, Dieterich DT, Samo T, Benson CA, et al. A randomized controlled study of intravenous ganciclovir therapy for cytomegalovirus peripheral retinitis in patients with AIDS. J Infect Dis 1993;168:557-63.

2. Foscarnet-Ganciclovir Cytomegalovirus Retinitis Trial. 4. Visual outcomes. Studies of Ocular Complications of AIDS Research Group in collaboration with the AIDS. Clinical Trials Group 1994;101:1250-61.

3. Palestine AG, Polis MA, De Smet MD, Baird BF, Falloon J, Kovacs $\mathrm{JA}$, et al. A randomized, controlled trial of foscarnet in the treatment of cytomegalovirus retinitis in patients with AIDS. Ann Intern Med 1991;115:665-73.

4. Lalezari JP, Stagg RJ, Kuppermann BD, Holland GN, Kramer F, Ives DV, et al. Intravenous cidofovir for peripheral cytomegalovirus retinitis in patients with AIDS, a randomized, controlled trial. Ann Intern Med 1997;126:257-63.

5. Parenteral cidofovir for cytomegalovirus retinitis in patients with AIDS: the HPMPC peripheral cytomegalovirus retinitis trial. A randomized, controlled trial. Studies of Ocular Complications of AIDS Research Group in Collaboration with the AIDS Clinical Trials Group. Ann Intern Med1997;126:264-74.

6. Drugs.com. Auckland. Drugsite Trust online; 2009. Available from: http://www.drugs.com/pro/valcyte.html. [Last accessed on 20 Nov 2009.

7. Rockville MD. US department of health and human services online. Available from: http://www.aidsinfo.nih.gov/DrugsNew/DrugDetailNT.aspx? MenuItem=DrugsandSearch=Onandint_id $=271$. [Last accessed on 20 Nov 2009]
8. Mark DP, John R, Robert MM, Carlos VP, John P, Freeman RB, et al. Valganciclovir results inimproved oral absorption of ganciclovir in liver transplant recipients. Antimicrob Agents Chemother 2000;4410:2811-5.

9. Ganapathy ME, Huang W, Wang H, Ganapathy V, Leibach FH. Valacyclovir: a substrate for the intestinal and renal peptide transporters PEPT1 and PEPT2. Biochem Biophys Res Commun 1998;2462:470-5.

10. Sinko PJ, Balimane PV. Carrier-mediated intestinal absorption of valacyclovir, the L-valyl ester prodrug of acyclovir: 1. Interactions with peptides, organic anions and organic cations in rats. Biopharm Drug Dispos 1998;194:209-17.

11. Aparna N, Srinivas Reddy P, Ramachary DB, Rajagopal S. Unraveling the stability of plasma proteins upon interaction of synthesized androstenedione and its derivatives-a biophysical and computational approach. ACS Omega 2017;2:6514-24.

12. Subramanyam R, Gollapudi A, Bonigala P, Chinnaboina M, Amooru DG. Betulinic acid binding to human serum albumin: a study of protein conformation and binding affinity. J Photochem Photobiol B 2009;94:8-12.

13. Morris GM, Huey R, Lindstrom W, Sanner MF, Belew RK, Goodsell DS, et al. AutoDock4 and AutoDockTools4: automated docking with selective receptor flexibility. J Comput Chem 2009;30:2785-91.

14. Xu H, Yao N, Xu H, Wang T, Li G, Li Z. Characterization of the interaction between eupatorin and bovine serum albumin by spectroscopic and molecular modeling methods. Int J Mol Sci 2013;14:14185-203.

15. Shi JH, Pan DQ, Wang XX, Liu TT, Jiang $M$, Wang $Q$. Characterizing the binding interaction between antimalarial artemether (AMT) and bovine serum albumin (BSA): spectroscopic and molecular docking methods. J Photochem Photobiol B 2016;16:14-23.

16. Lakowicz JR, Principles of fluorescence spectroscopy. 2nd ed. Plenum Press; New York: 1999. p. 237.

17. Nooshin B, Yalda S, Mohammad Reza AK, Samira R, Shahram P, Maryam MA, et al. Spectroscopic study of the interaction between osthole and human serum albumin: identification of the possible binding site of the compound. J Lumin 2013;143:328-36.

18. Yuan T, Weljie AM, Vogel HJ. Tryptophan fluorescence quenching by methionine and selenomethionine residues of calmodulin: orientation of peptide and protein binding. Biochemistry 1998;37:3187-95.

19. Fuller CW, Miller JN. Silver medal lectures Proc Anal Div Chem Soc 1979;16:199-8.

20. Tang JH, Luan F, Chen XG. Binding analysis of glycyrrhetinic acid to human serum albumin: Fluorescence spectroscopy, FTIR, and molecular modeling. Bioorg Med Chem 2006;14:3210-17.

21. Ross PD, Subramanian S. Thermodynamics of protein association reactions: forces contributing to stability. Biochemistry 1981;20:3096-102.

22. Yamasaki K, GChuang VT, Maruyama T, Otagiri M. Albumindrug interaction and its clinical implication. Biochim Biophys Acta 2013;1830:5435-43.

23. Sudlow G, Birkett DJ, Wade DN. The characterization of two specific drug binding sites on human serum albumin. Mol Pharmacol 1975;11:824-32.

24. Xu H, Liu QW, Wen YQ. Spectroscopic studies on the interaction between nicotinamide and bovine serum albumin. Spectrochim Acta Part A 2008;71:984-8.

25. Yeggoni DP, Subramanyam R. Binding studies of L-3,4dihydroxyphenylalanine with human serum albumin. Mol Biosyst 2014;10:3101-10.

26. Ramachander M, Kalpana VS. Molecular docking of amitriptyline to ceruloplasmin, retinol-binding protein and serum albumin. Asian J Pharm Clin Res 2018;11:169-75. 